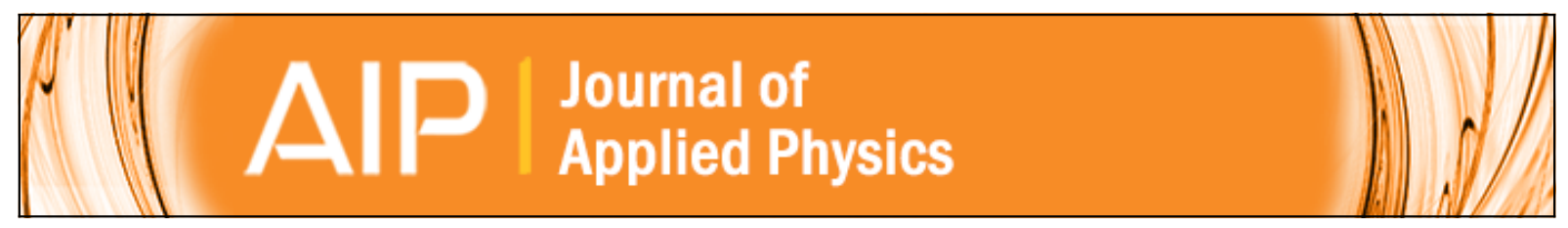

\title{
Spontaneous oxidation of disordered fcc FePt nanoparticles
}

P. de la Presa, T. Rueda, A. Hernando, J. M. Ramallo-López, L. J. Giovanetti, and F. G. Requejo

Citation: Journal of Applied Physics 103, 103909 (2008); doi: 10.1063/1.2931947

View online: http://dx.doi.org/10.1063/1.2931947

View Table of Contents: http://scitation.aip.org/content/aip/journal/jap/103/10?ver=pdfcov

Published by the AIP Publishing

\section{Articles you may be interested in}

Influence of excess Fe accumulation over the surface of FePt nanoparticles: Structural and magnetic properties J. Appl. Phys. 113, 134303 (2013); 10.1063/1.4796091

Coercive field and energy barriers in partially disordered FePt nanoparticles

J. Appl. Phys. 105, 07B514 (2009); 10.1063/1.3067570

Magnetic assembles of FePt (001) nanoparticles with Si O 2 addition

J. Appl. Phys. 105, $07 A 715$ (2009); 10.1063/1.3058690

X-ray absorption and diffraction studies of thin polymer/FePt nanoparticle assemblies

J. Appl. Phys. 93, 6299 (2003); 10.1063/1.1567802

Coercivity and remanence in self-assembled FePt nanoparticle arrays

J. Appl. Phys. 93, 7041 (2003); 10.1063/1.1557398

\section{AlP $\left.\right|_{\text {APL Photonics }}$}

APL Photonics is pleased to announce Benjamin Eggleton as its Editor-in-Chief

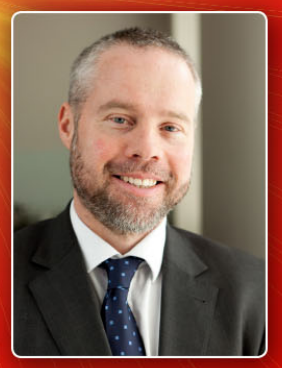




\title{
Spontaneous oxidation of disordered fcc FePt nanoparticles
}

\author{
P. de la Presa, ${ }^{1, a)}$ T. Rueda, ${ }^{1}$ A. Hernando, ${ }^{1}$ J. M. Ramallo-López, ${ }^{2}$ L. J. Giovanetti, ${ }^{2}$ \\ and F. G. Requejo ${ }^{2}$ \\ ${ }_{1}^{1}$ Instituto de Magnetismo Aplicado, UCM-ADIF-CSIC, P.O. Box 155, Las Rozas, Madrid, E-28230 Spain \\ ${ }^{2}$ INIFTA and IFLP, CONICET and Facultad de Ciencias Exactas, Universidad Nacional de La \\ Plata, P.O. Box 16, Suc. 4, 1900 La Plata, Buenos Aires, Argentina
}

(Received 29 November 2007; accepted 23 March 2008; published online 28 May 2008)

\begin{abstract}
In this work we present new results on spontaneous oxidation of disordered fcc FePt nanoparticles. The "as-made" oleic acid and oleylamine coated FePt nanoparticles of average size $4 \mathrm{~nm}$ synthesized by a high-boiling coordinating solvent method were exposed to air over a period of days and characterized structurally and magnetically by means of different techniques such as XANES, XPS, EXAFS, and SQUID magnetometry. The "as-made" FePt nanoparticles stabilize in the disordered fcc structure and have a very low magnetic saturation $\left(\mathrm{M}_{s}=11 \mathrm{emu} / \mathrm{g}\right)$ and a huge coercive field $\left(\mathrm{H}_{c}=1800 \mathrm{Oe}\right)$ compared to the low temperature bulk values of the disordered fcc FePt. We observed that the coercive field and the magnetic saturation change with the time the sample is exposed to air and these changes are associated with the oxidation or passivation of the nanoparticle surface that gives place to a core-shell structure. Indeed, the study on the electronic properties of the nanoparticles confirms the magnetic results and indicates that when the nanoparticles are exposed to air, changes in the oxidation state of both $\mathrm{Fe}$ and $\mathrm{Pt}$ occur, the oxidation state of Fe coming close to hematite. The formation of hematite tends to soften the "as-made" FePt nanoparticles as observed by the reduction of the coercive field to almost one third of the original value. Although the hematite softens the FePt nanoparticles, there is an exchange coupling at the interface of the core-shell characterized by the increase of the coercive field from 300 to 900 Oe when the sample is cooled in an applied field of $50 \mathrm{kOe}$. (C) 2008 American Institute of Physics.
\end{abstract}

[DOI: $10.1063 / 1.2931947]$

\section{INTRODUCTION}

It is often assumed that FePt is resistant to oxidation in air even in the nanometric range, at least in the equiatomic composition, contrasting with pure metal $\mathrm{Fe}$, $\mathrm{Co}$, and $\mathrm{Ni}$ nanoparticles (NPs) of the same size, which are in order of milliseconds covered by an oxide shell. ${ }^{1}$ Apparently, the presence of Pt would contribute to the inertness of the alloy, resulting in a material that, depending on the stabilized structure, is well appropriated for biomedical or technological applications. ${ }^{2}$ However, there are evidences that belie the premise the $\mathrm{FePt}$ is resistant to oxidation in the nanometer scale. In a recent work, Delalande et $a l .{ }^{3}$ have shown that in four different syntheses by high-boiling coordinating solvent method, the so-called "hot-soap method," the as-made FePt NPs $4 \mathrm{~nm}$ in size have a rich Pt core and nonmagnetic $\mathrm{Fe}_{\text {III }}$ located at the surface, forming a core-shell structure. Möessbauer results recently published indicate that the shell is made up of distorted spinel-type iron oxides like maghemite $\left(\gamma-\mathrm{Fe}_{2} \mathrm{O}_{3}\right){ }^{4}$ There exist also previous works that report on this core-shell structure of FePt nanoparticles. Wu et al. ${ }^{5}$ ascribe the low magnetization state of the as-made FePt NPs to the formation of a nonmagnetic shell (of about $0.5 \mathrm{~nm}$ ) arising from the bonding of the acid group of oleic acid to Fe. A very thin oxide layer $(0.4 \mathrm{~nm})$ surrounding a FePt core has been also detected by near edge $\mathrm{x}$-ray absorption fine

\footnotetext{
${ }^{a)}$ Electronic mail: pdelapresa@adif.es.
}

structure (NEXAFS). ${ }^{6}$ Evidence of about $20 \%$ of nonmetallic Fe in the as-made FePt NPs has been reported by Stahl et $_{\text {al. }}{ }^{7}$

These results all have some common features: (i) the NPs have been synthesized by hot-soap methods, (ii) they are about $4 \mathrm{~nm}$ in size with small size distribution, (iii) the saturation magnetization $\left(\mathrm{M}_{s}\right)$ is much lower than expected, (iv) at room temperature (RT), well above the blocking temperature $\mathrm{T}_{B}$, it is rather unusual to observe hysteresis loops following the Langevin law for superparamagnetic NPs, and (v) although the FePt NPs synthesize in the disordered fcc structure, they have, surprisingly, a relatively high magnetic anisotropy at low temperature (about $10^{5} \mathrm{~J} / \mathrm{m}^{3}$ ).

Depending on the magnetic properties of the core and of the shell, a core-shell structure can originate exchange coupling at the interface. Since the exchange coupling of magnetically hard and soft phases may greatly enhance the energy products of the two phases, the fabrication of exchange coupled nanocomposites using nanoparticle self-assembled is a great challenge. ${ }^{8,9}$ Many approaches have been done in this way. For example, annealing of binary assemblies of $\mathrm{FePt}$ and $\mathrm{Fe}_{3} \mathrm{O}_{4}$ particles converts the assembly into $\mathrm{FePt}-\mathrm{Fe}_{3} \mathrm{Pt}$ nanocomposites, with $\mathrm{FePt}$ and $\mathrm{Fe}_{3} \mathrm{Pt}$ magnetically hard and soft phases, respectively. The exchange coupling can be tuned by tuning the size and composition of the individual kind of NPs. ${ }^{8}$ Since natural oxidation of FePt NPs gives place to a core-shell structure, it is of interest to study the magnetic properties of this core-shell.

In this work we synthesize fcc FePt NPs of about $4 \mathrm{~nm}$ in size by high-boiling coordinating solvent method and 
study the changes in the electronic and magnetic properties when the NPs are exposed to air. The oxidation states and the changes originated by the air exposure of $\mathrm{Fe}$ and $\mathrm{Pt}$ are in-

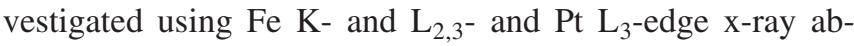
sorption near edge spectroscopy (XANES), $\mathrm{Pt} \mathrm{L}_{3}$-edge extended $\mathrm{x}$-ray absorption fine structure (EXAFS), and x-ray photoelectron spectroscopy (XPS). The air exposure bears also on $\mathrm{M}_{s}$ and on the coercive field $\left(\mathrm{H}_{c}\right), \mathrm{H}_{c}$ being dramatically reduced. We show that the exposition to air leads to an oxidation process that evolves with the course of the time. Our results are consistent with the formation of an oxide shell and we also show that in the naturally oxidized FePt there exists an exchange coupling at the interface of the ferromagnetic core and the oxidized shell when the NPs are cooled down in an applied field of $50 \mathrm{kOe}$.

\section{EXPERIMENTAL DETAILS}

\section{A. Sample preparation}

The FePt NPs have been synthesized by one of the hotsoap methods, the protocol of Sun et al. ${ }^{10}$ The procedure involved heating $0.5 \mathrm{mmol}$ of $\mathrm{Pt}(\mathrm{acac})_{3}$ in $20 \mathrm{ml}$ of dioctylether in an inert $\mathrm{N}_{2}$ atmosphere to $100{ }^{\circ} \mathrm{C}$, followed by the injection of $0.5 \mathrm{mmol}$ each of oleic acid and oleylamine and $1 \mathrm{mmol}$ of $\mathrm{Fe}(\mathrm{CO})_{5}$. The solution was further heated up to the boiling point of dioctylether and was refluxed for 30 min. After that it was cooled down to room temperature (RT) under $\mathrm{N}_{2}$ atmosphere. The FePt nanoparticles were cleaned by cyclic precipitation and redispersion with ethanol and hexane, respectively. Finally, the particles were stored in hexane with oleic acid and oleylamine.

Before each magnetic characterization, the NPs were taken from the dispersion, precipitated with ethanol and centrifuged, the supernatant was discarded, and the sample was dried at RT in vacuum ("freshly precipitated"). The black powder was weighed on an analytical balance and put in a glycerin capsule to perform the magnetic measurements (batch I). Another part of the sample was precipitated from the dispersion, dried at $50{ }^{\circ} \mathrm{C}$ during $15 \mathrm{~min}$ at air (batch II), and kept in the glycerin capsule to be measured. For the structural and the electronic characterization, the freshly precipitated NPs were set in the sample holder in air and measured. No special care was taken for the manipulation of the sample.

\section{B. Structural characterization}

The sample was measured by $\mathrm{x}$-ray diffraction in a range from $30^{\circ}$ to $70^{\circ}$ with a step of $0.02^{\circ}$. Analysis of the asprepared nanoparticles reveals the characteristic diffraction pattern of the fcc FePt. A size of $3 \mathrm{~nm}$ was estimated by means of the Scherrer formula. No traces of Fe oxides were detected at least in the resolution of the equipment.

Particle size was determined from transmission electron microscopy (TEM) micrographs in a $200 \mathrm{keV}$ JEOL-2000 FXII microscope. For the observation of the sample in the microscope, the particles were dispersed in hexane and a drop of the suspension was placed onto a copper grid covered by a carbon film. The NPs are rather spherical in shape and have a mean diameter of $4 \mathrm{~nm}$ with a narrow size

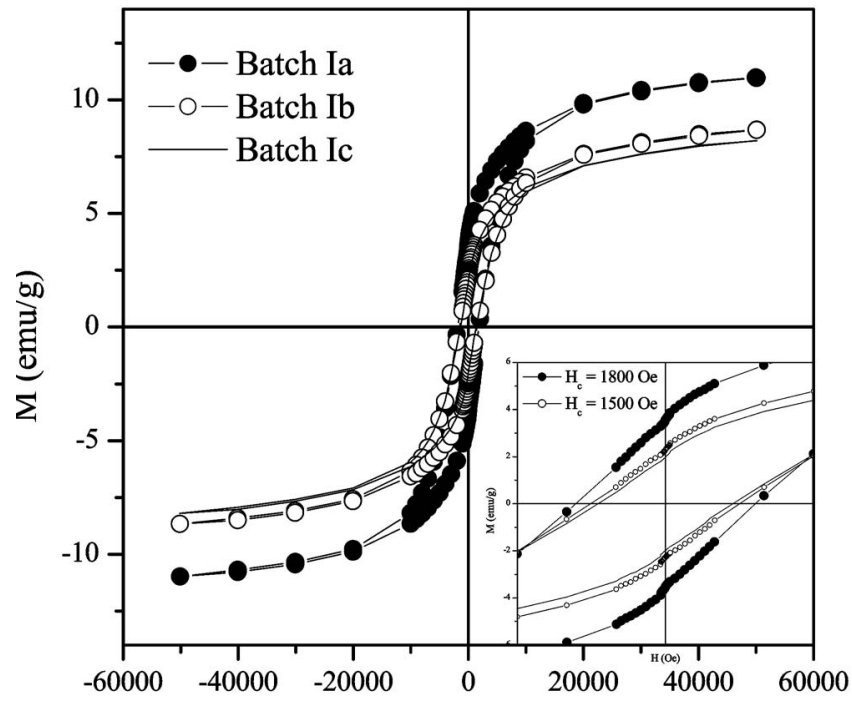

FIG. 1. Magnetic curve at $5 \mathrm{~K}$ of the as-prepared sample (batch Ia), 15 days later (batch $\mathrm{Ib}$ ), and 45 days later (batch Ic). The inset shows the coercive fields in detail.

distribution. ${ }^{11}$ Energy dispersive $\mathrm{x}$-ray (EDX) analysis yields a mean composition of $\mathrm{Fe}_{45} \mathrm{Pt}_{55}$ in the $\mathrm{Pt}$ rich side.

\section{XANES, XPS, and EXAFS measurements}

The Fe $\mathrm{L}_{2,3}$-edge XANES spectra were measured by total electron yield (TEY) at the 9.3.2 beam line at the Advanced Light Source (ALS, Berkeley, CA). The experiments were carried out using a grating of 600 lines $/ \mathrm{mm}$ and a slit aperture that gives a resolution of $\mathrm{E} / \Delta \mathrm{E}=3000$. Normalized XANES spectra were obtained after the subtraction of a linear background obtained by fitting the $\mathrm{Fe}_{3}$ pre-edge region. The normalization was carried out by averaging the linear region between 726 and $728 \mathrm{eV}$. Pt $4 f$ XPS experiments were also performed at beam line 9.3.2 using $600 \mathrm{eV}$ incident photon energy. Fe $\mathrm{K}$ and $\mathrm{Pt}_{2}$ XANES and $\mathrm{Pt} \mathrm{L}_{3}$ EXAFS experiments were performed at the XAS-2 beamline at the Laboratorio Nacional de Luz Sincrotron (Campinas, Brazil) at room temperature in transmission mode using a $\mathrm{Si}$ (111) crystal as monochromator. The EXAFS signals were extracted from the absorption spectra using the Athena program and analyzed using the Arthemis program. ${ }^{12}$ Phase and amplitude of reference compounds used in the analysis were generated using the FEFF code. ${ }^{13}$

\section{Magnetization measurement}

The FePt NPs have been magnetically characterized by means of a Quantum Design SQUID magnetometer. The magnetic characterization consists in magnetization cycles in a maximum applied field of $50 \mathrm{kOe}$ and zero-field-cooled (ZFC) and field-cooled (FC) curves. Batch I was measured immediately after precipitation of the NPs from the dispersion and drying them in vacuum (batch Ia); the sample was maintained in the glycerin capsule in order to evaluate if, after some time, there exist changes in the sample but the mass and the type of NPs are preserved. The following measurement was performed 15 and 45 days later (batches Ib and Ic, respectively) at $5 \mathrm{~K}$ (see Fig. 1). 


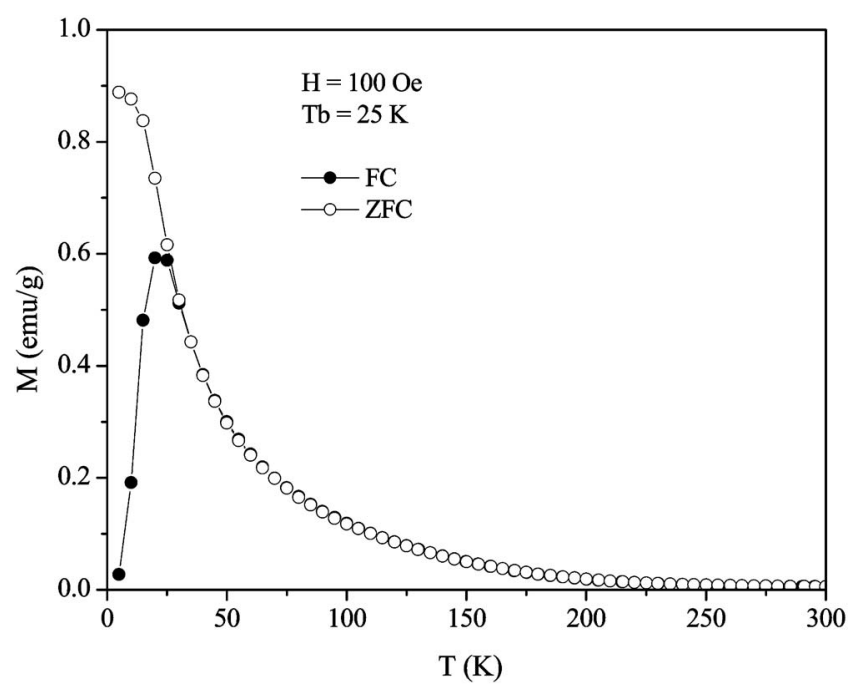

FIG. 2. ZFC (open symbols) and FC (full symbols) curves as a function of temperature and at external field $\mathrm{H}=100$ Oe for batch Ia.

Magnetization curves were measured between 5 and 300 $\mathrm{K}$ in an applied field of 100 Oe after cooling the sample in zero magnetic field and after cooling in an external magnetic field of 100 Oe (see Fig. 2). The ZFC-FC magnetization curves show the behavior of super-paramagnetic nanoparticles: the ZFC curve has a maximum at $25 \mathrm{~K}$ whereas the FC curve decreases with increasing temperature. At $30 \mathrm{~K}$ both curves converge and show the same temperature dependence for increasing temperatures, indicating a regime of super-paramagnetism.

Batch II was dried at $50{ }^{\circ} \mathrm{C}$ for $15 \mathrm{~min}$; after that, cycles at 5 and $300 \mathrm{~K}$ (batch IIa) were performed. It is known that at $50{ }^{\circ} \mathrm{C}$ the organic surfactant does not carbonize although it degrades; therefore, the temperature acts as a catalyst of the surface degradation. The sample was kept in the glycerin capsule and new cycles at $5 \mathrm{~K}$ were measured 20, 30, and 45 days later (batches IIb, IIc, and IId, respectively); no noticeable changes were observed between batches IIc and IId (see Fig. 3; for the sake of clarity we do not show the magnetic loop of batch IId).

$\mathrm{ZFC}$ and $\mathrm{FC}$ hysteresis loops at $5 \mathrm{~K}$ were measured in batches IIc and IId. In the FC procedure, the sample was cooled down from RT to $5 \mathrm{~K}$ under an applied field of 50 $\mathrm{kOe}$. Once the temperature was reached, the measurement of the loop started (see Fig. 4).

\section{RESULTS}

\section{A. Electronic characterization}

Figure 5 shows the normalized $\mathrm{Fe} \mathrm{L}_{2,3}$-edge XANES spectra. The measurements were performed in ultra high vacuum conditions. Figure 5(a) corresponds to the XANES spectrum of the NPs freshly precipitated and set in the sample holder. Then, the NPs were exposed for 20 days to air and a new XANES characterization was performed. As can be seen from Fig. 5(b), the shape of the spectra has changed and draws near to the hematite one [Fig. 5(c)], indicating that

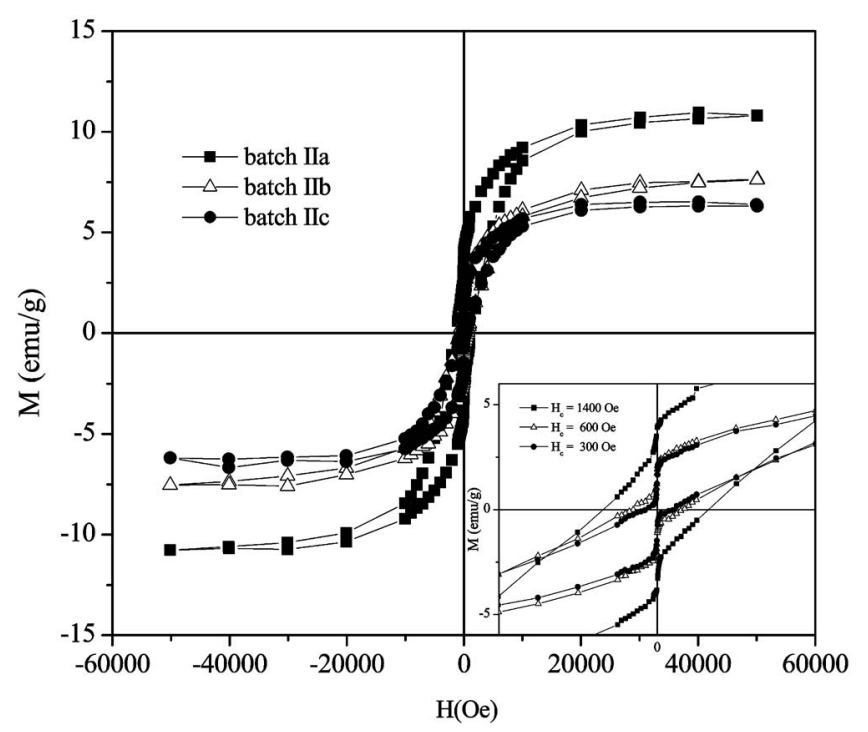

FIG. 3. Magnetic curve at $5 \mathrm{~K}$ of the sample heated at $50{ }^{\circ} \mathrm{C}$ for $15 \mathrm{~min}$ (batch IIa), 20 days later (batch IIb), and 30 days later (batch IIc). The inset shows the coercive fields in detail.

the average oxidation state of the Fe in the FePt NPs increases with respect to the first measurement (i.e., FePt NPs freshly precipitated).

In order to characterize the oxidation state of $\mathrm{Fe}$ in the naturally oxidized sample, we perform a Fe K-edge XANES measurement of FePt NPs. Figure 6(a) shows the Fe K-edge spectrum in FePt NPs as well as those belonging to several Fe-reference compounds. By visual inspection of the spectrum, the Fe in the FePt NPs is not in pure metallic state; even more, the Fe is highly oxidized. A quantitative result can be obtained by calculation of the energy shift with respect to the metallic state [see Fig. 6(b)]. According to this procedure, the average oxidation state for $\mathrm{Fe}$ is 2.7.

The XANES spectra of Pt $\mathrm{L}_{3}$-edge (Fig. 7) show also differences that depend on the measurement conditions. As the sample is taken out of the dispersion, the white line likens to the Pt bulk [see Fig. 7(a)]. However, after some days of air exposure, the white line of FePt NPs changes and becomes higher than the Pt bulk one, as shown in Fig. 7(b).

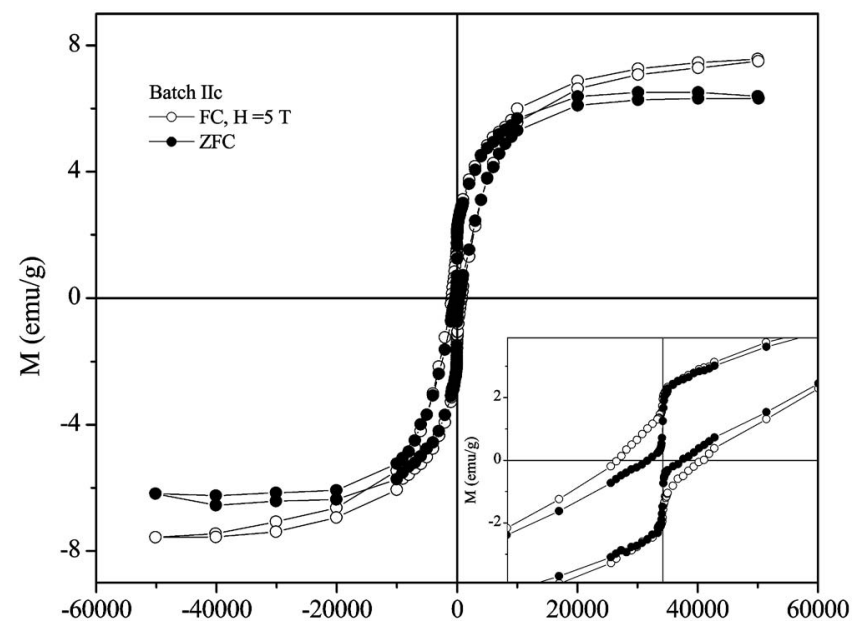

FIG. 4. ZFC-FC hysteresis loop at $5 \mathrm{~K}$ with external field $50 \mathrm{kOe}$. 


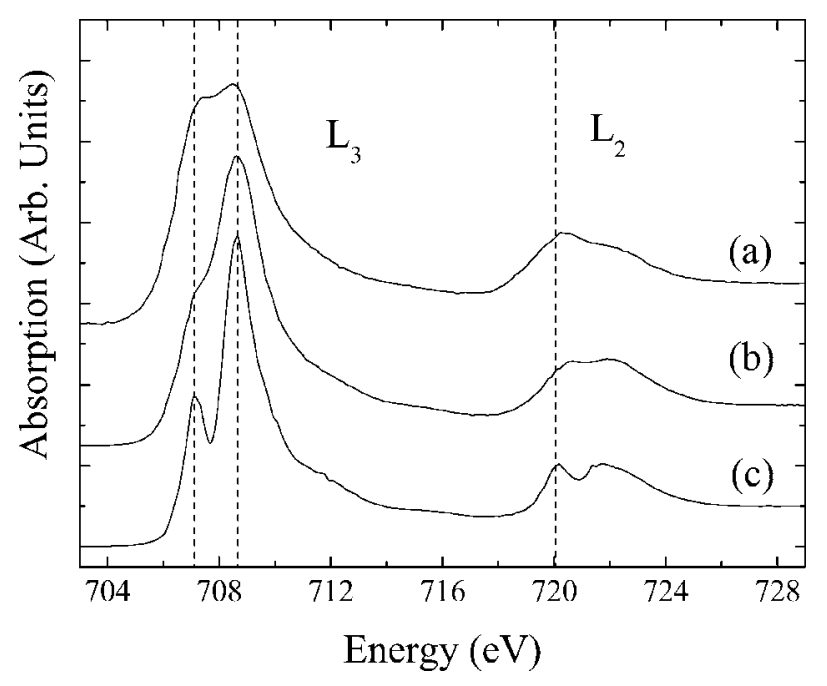

FIG. 5. Fe $\mathrm{L}_{2,3}$ XANES of samples (a) freshly precipitated, (b) after 20 days exposed to air, and (c) hematite used as reference. Vertical lines indicate the position of the resonance peaks in the hematite reference.

Figure 8 shows the Pt $4 f$ XPS spectra of samples freshly precipitated (a) and exposed to air (b). Figure 8(a) shows the expected splitting for metallic Pt $4 f$ level at 74.6 and $71.2 \mathrm{eV}$ for $4 f_{5 / 2}$ and $4 f_{7 / 2}$ electronic levels, respectively. The fit performed in the sample exposed to air [Fig. 8(b)] shows the contribution of Pt oxide at 75.3 and $72.0 \mathrm{eV}$. This is also
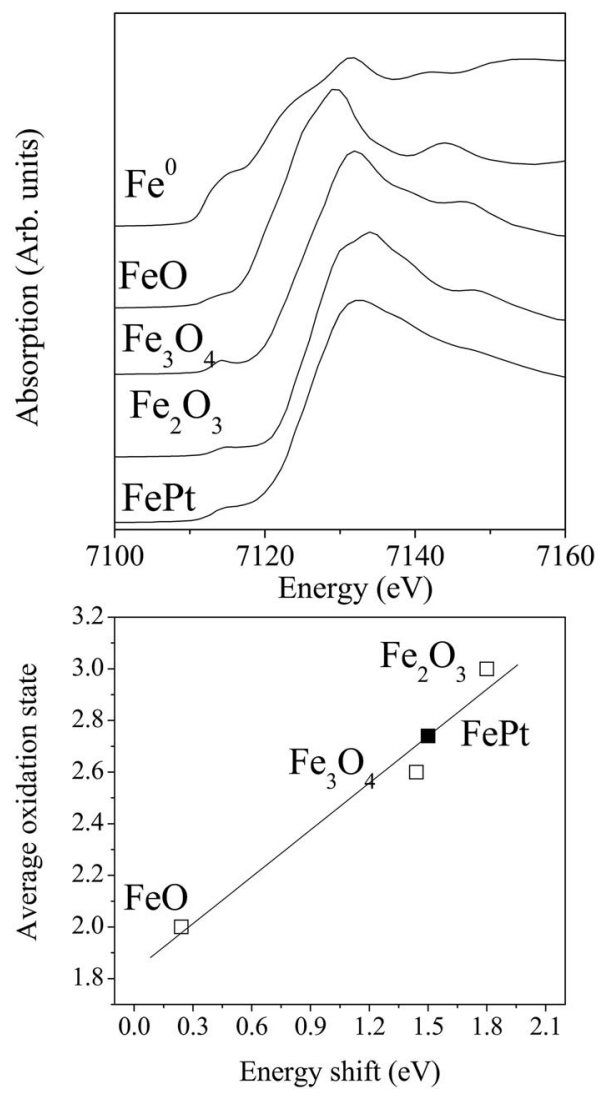

FIG. 6. (a) XANES spectra at the Fe K-edge of several Fe reference compounds and FePt nanoparticles in pellet. (b) Oxidation state determination of Fe compounds obtained employing the Fe K-edge energy shift. The corresponding energy shift for pure metallic Fe foil was taken as reference (zero energy shifting for zero oxidation state). Fe oxidation state in FePt is determined from the linear fit.

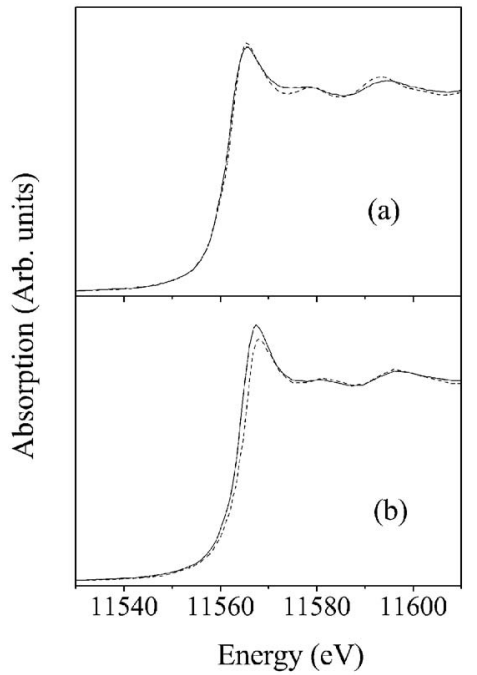

FIG. 7. XANES spectra at the $\mathrm{Pt}_{2}$-edge of FePt NP (a) in pellet after some minutes of air exposure and (b) in pellet after some days of air exposure. XANES spectra of metallic Pt are also shown (dashed line) for comparison.

shown by the $\mathrm{Pt}_{3}$ white line increment at the $\mathrm{Pt} \mathrm{L}_{3}$ XANES spectra (see Fig. 7). A rough estimation of the electron effective-attenuation-length ${ }^{14}$ suggests that the electrons collected in the Pt $4 f$ XPS experiment with a kinetic energy of $530 \mathrm{eV}$ come from a depth lower than $0.7 \mathrm{~nm}$.

Figures 9(a) and 9(b) show the Fourier transform of EXAFS at the Pt $\mathrm{L}_{3}$ signal in FePt NPs with different fit proposals. Considering the fit with only Pt-Pt shells [Fig. 9(a)], the average coordination number $N$ for Pt-Pt is consistent with the existence of $\mathrm{Pt}$ metallic clusters. The fit results clearly improved [Fig. 9(b)] when a second coordination sphere of $\mathrm{Fe}$ atoms is proposed (see Table I). In addition, the fact that the average coordination number $N$ is about 1.3 indicates the small content of FePt alloy in the NP, consistent with Fe K XANES results. Figure 9(c) shows the Fourier transform of the Fe K EXAFS signal.

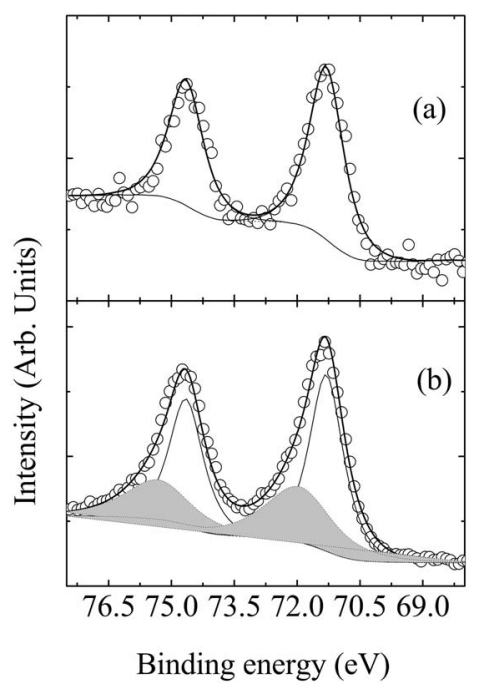

FIG. 8. XPS Pt $4 f$ spectra taken at $600 \mathrm{eV}$ for incident photon energy of samples (a) freshly precipitated FePt NPs and (b) FePt NP exposed to air. The sample exposed to air clearly shows the appearance of a Pt oxide contribution. 


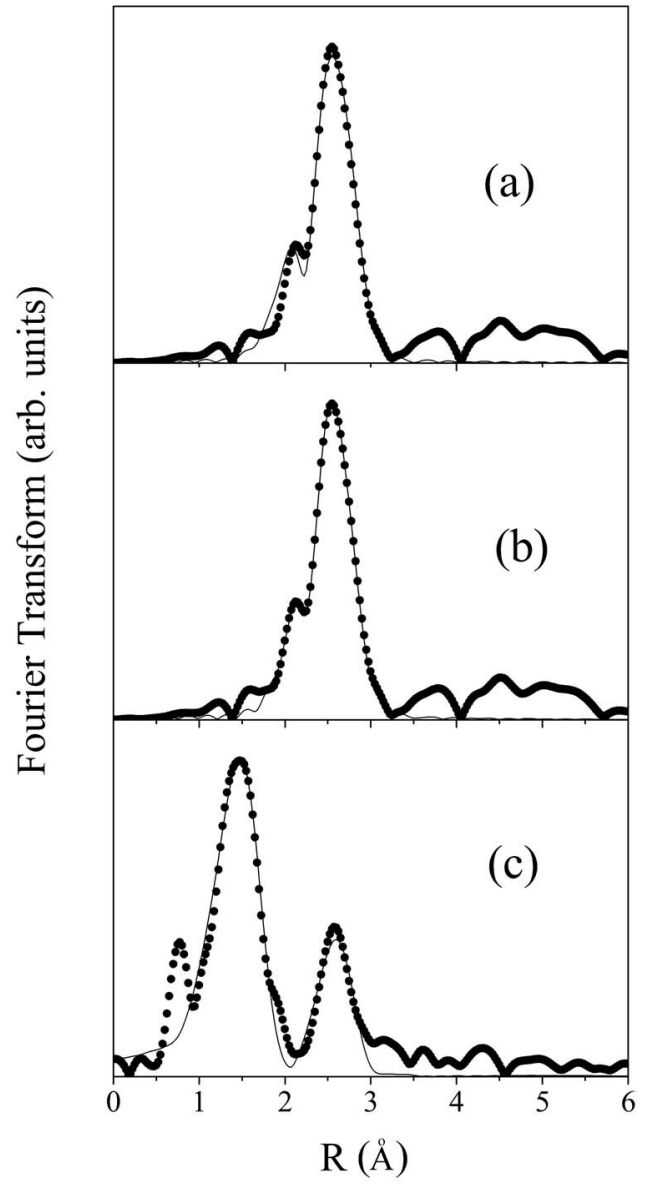

FIG. 9. Fourier transform of the EXAFS signal at the $\mathrm{Pt}_{3}$-edge of $\mathrm{PtFe}$ NPs (filled circles), solid lines show the fitted function considering (a) only Pt scatterers and (b) both Pt and Fe scatterers in the surroundings of the absorbing atom. (c) Fourier transform of the EXAFS signal at the Fe K-edge of FePt NP (filled dots). Solid line shows the fitted function.

\section{B. Magnetic properties}

As can be seen from Fig. 1, the freshly precipitated sample (batch Ia) has $\mathrm{H}_{c}=1800$ Oe and $\mathrm{M}_{s} \approx 11 \mathrm{emu} / \mathrm{g}$ (per gram of $\mathrm{FePt}$ ). The $\mathrm{M}$ versus $\mathrm{H}$ cycle was repeated after the 15 days the sample was kept in the glycerin capsule. As a result the saturation is reduced to $\approx 9 \mathrm{emu} / \mathrm{g}$ and $\mathrm{H}_{c}$ decreases to $1500 \mathrm{Oe}$ (batch $\mathrm{Ib}$ ). Since the mass and the type of the NPs remain the same, we can assume that the decrease of $\mathrm{M}_{s}$ is due to the formation of a surface layer that changes its magnetic properties, becoming nonmagnetic or reducing the magnetic contribution. If this surface layer is nonmagnetic, a first approximation of the thickness of the layer can be done. Assuming that the density of the magnetic part does not change, a layer of about $0.3 \mathrm{~nm}$ thickness of nonmagnetic

TABLE I. EXAFS parameters for the fitted functions shown in Fig. 9.

\begin{tabular}{lccccc}
\hline \hline Fit & Scatterer & $\mathrm{N}$ & $\mathrm{R}(\AA)$ & $\sigma^{2}\left(\AA^{-2}\right)$ & $\mathrm{E}_{0}(\mathrm{eV})$ \\
\hline $\mathrm{a}$ & $\mathrm{Pt}$ & $10.7 \pm 1$ & $2.72 \pm 0.02$ & $0.008 \pm 0.002$ & $-3.8 \pm 0.5$ \\
$\mathrm{~b}$ & $\mathrm{Fe}$ & $1.3 \pm 0.5$ & $2.67 \pm 0.02$ & $0.007 \pm 0.001$ & $1.7 \pm 0.3$ \\
& $\mathrm{Pt}$ & $6.3 \pm 1$ & $2.74 \pm 0.02$ & $0.006 \pm 0.001$ & $1.7 \pm 0.3$ \\
$\mathrm{c}$ & $\mathrm{O}$ & $5 \pm 1$ & $1.97 \pm 0.02$ & $0.011 \pm 0.002$ & $-1.5 \pm 0.1$ \\
& $\mathrm{Fe}$ & $1.7 \pm 0.4$ & $3.02 \pm 0.02$ & $0.007 \pm 0.001$ & $-1.5 \pm 0.1$ \\
\hline \hline
\end{tabular}

contribution can reduce the $\mathrm{M}_{s}$ from 11 to $9 \mathrm{emu} / \mathrm{g}$, and the FePt NP can be considered as formed by a magnetic nucleus of about $3.4 \mathrm{~nm}$ and a dead layer of $0.3 \mathrm{~nm}$, which agrees well with the values reported by other authors. ${ }^{5,6}$ It is worth mentioning that the most important changes occur during the first 15 days; there were no noticeable changes after 45 days (batch Ic).

At $5 \mathrm{~K}$ the value of remanence in batch Ia is close to the value $0.3 \mathrm{M}_{s}$, below the expected value at $0 \mathrm{~K}$ for an assembly of non-interacting, uniaxial single-domain particles with randomly oriented axis. The surfactants oleic acid and oleylamine contain 18 carbon units, providing a separation between the particles of at least $3 \mathrm{~nm}$ when the NPs are dispersed in the solvent or dried on a substrate. At these distances, magnetic dipolar interactions are weak. However, when the NPs are loaded into the glycerin capsules they are pressed in order to avoid movements of the NPs when they are set in a magnetic field. Then, the distance between particles decreases, the magnetic dipolar interaction cannot be neglected, and, consequently, the system cannot be considered as non-interacting. Therefore, deviations from the noninteracting case are expected in our results.

The magnetization curves measured for batch Ia as a function of temperature in ZFC-FC procedure in an applied field of 100 Oe (Fig. 2) show the typical process of an assembly of super-paramagnetic particles with distribution of blocking temperatures. Assuming a spherical shape of the particles with a mean volume $V$, the effective anisotropy constant can be estimated from $\mathrm{K}_{\text {eff }} \approx 25 k_{B} T_{B} / V$, with $k_{B}$ the Boltzmann constant and $T_{B}$ the measured blocking temperatures, giving an effective anisotropy value $\approx 6 \quad 10^{5} \mathrm{~J} / \mathrm{m}^{-3}$. Although the calculation for the effective anisotropy is for non-interacting NPs, we can assume that the obtained value is a good estimation of $\mathrm{K}_{e f f}$, at least in first approximation.

Considering the changes in the magnetic properties under air exposure, we perform a mild thermal treatment at $50{ }^{\circ} \mathrm{C}$ for $15 \mathrm{~min}$ in order to accelerate the degradation of organic coat but avoid an induced oxidation. The sample was immediately measured in the SQUID (batch IIa). The magnetic moment at $50 \mathrm{kOe}$ does not show noticeable changes with respect to batch Ia, but we can observe two main differences: (i) $\mathrm{H}_{c}=1400$ Oe is smaller than in batch Ia and (ii) there is a steep decrease of the magnetization around zero external field (see Fig. 3). Due to the steep decrease of magnetization at zero external field, the magnetic curve of batch IIa looks like the sum of two non-interacting magnetic phases: a hard and a soft phase; however, the kink at around zero field is a signal that either there are NPs still not blocked at $5 \mathrm{~K}$ (as proposed by Stahl et al. ${ }^{7}$ ) or the surface of the NPs oxidizes. As can be seen in Fig. 3, the steep decrease of magnetization becomes steepest after 20 days and still increases 30 days later (after 45 days it remains almost unchanged). This is an indication that points to an oxidation process rather than the contribution of unblocked NPs.

In batches Ia, Ib, and Ic we do not observe the kink at zero external field, although both, $\mathrm{M}_{s}$ at $50 \mathrm{kOe}$ and $\mathrm{H}_{c}$, decrease after 15 days of air exposure.

The $\mathrm{H}_{c}$ decreases from 1400 Oe for the as prepared sample (batch IIa), to 600 Oe for the sample measured 20 
TABLE II. The table shows the time the NPs are exposed to air $\mathrm{t}_{e}$, the coercive fields $\mathrm{H}_{c}$, and the magnetic saturations $\mathrm{M}_{s}$.

\begin{tabular}{lccc}
\hline \hline Batches & $\mathrm{t}_{e}($ days $)$ & $\mathrm{H}_{c}(\mathrm{Oe})$ & $\mathrm{M}_{s}(\mathrm{emu} / \mathrm{g})$ \\
\hline IIa & 0 & 1400 & 10.8 \\
IIb & 20 & 600 & 7.6 \\
IIc & 30 & 300 & 6.5 \\
IId & 45 & 300 & 6.3 \\
\hline \hline
\end{tabular}

days later (batch $\mathrm{IIb}$ ), and to 300 Oe for the sample measured 30 and 45 days later (batches IIc and IId). The magnetic moment at $5 \mathrm{~K}$ is also affected by the air exposure: the $\mathrm{M}_{s}$ at $5 \mathrm{~K}$ changes from 10.8 (batch IIa), to 7.6 (batch IIb), to $6.3 \mathrm{emu} / \mathrm{g}$ (batch IIc), and remains almost constant for batch IId. Table II resumes the main results of batch II. Since the large surface of the NPs is the mainly affected by the medium conditions, the changes in $\mathrm{H}_{c}$ and $\mathrm{M}_{s}$ and the increase of the kink are the consequences of the degradation of the NP surfaces exposed to air.

ZFC-FC hysteresis loops were performed in batches IIc and IId at $50 \mathrm{kOe}$ and $5 \mathrm{~K}$ (Fig. 4 shows the hysteresis loop in batch IIc). The ZFC magnetic curve at $5 \mathrm{~K}$ has $\mathrm{H}_{c}$ $=300 \mathrm{Oe}$ and $\mathrm{M}_{s}=6.3 \mathrm{emu} / \mathrm{g}$. When the sample is cooled down in a $50 \mathrm{kOe}$ external field and the hysteresis loop is measured up to $50 \mathrm{kOe}$, the coercivity increases to $900 \mathrm{Oe}$, and $\mathrm{M}_{s}=7.6 \mathrm{emu} / \mathrm{g}$. Batch IId shows very similar behavior. The changes in the coercivity and in the saturation reflect exchange interactions at the interface of a core-shell structure.

\section{DISCUSSION}

As the sample is exposed to air, the $\mathrm{Fe} \mathrm{L}_{2,3}$ XANES spectrum [see Fig. 5(b)] shows a combination of almost two different oxidation states. This intermediate oxidation state for Fe, between metallic and hematite "state," could be associated with the presence of metallic $\mathrm{Fe}$ and/or forming FePt alloy ${ }^{15}$ plus iron oxide. As obtained from the Fe K-edge XANES results, the average value of the oxidation state of $\mathrm{Fe}$ is 2.7 .

The Fourier transform of EXAFS at the Fe $\mathrm{K}$ - and $\mathrm{Pt}$ $\mathrm{L}_{3}$-edges signals in FePt NPs gives the average coordination number $N=1.3$, indicating the content of FePt alloy in the NP is small in agreement with Fe K XANES results. The majority of the Fe atoms are coordinated by oxygen atoms forming iron oxide. The obtained values suggest that only $15 \%$ of the Fe atoms could be bonded to Pt forming FePt alloy.

XANES spectra at the $\mathrm{Pt}_{3}$-edge (Fig. 7) clearly show the relative changes in the white line intensity of the sample with respect to the metallic state as the sample is exposed to air. As it can be seen from Fig. 7(a), the white line intensity of the nanoparticles freshly precipitated and that of the $\mathrm{Pt}$ bulk are similar. After some days, the behavior changes and the white line intensity of the nanoparticles is higher than that of the Pt bulk, indicating a decrease of the $5 d$ electrons occupancy, i.e., an increase in the number of holes at the same level. Several factors could be responsible for this behavior but the most probable can be attributed to the superficial oxidation of the Pt clusters in the NP.

The XPS results at the Pt $4 f$ level give a hint. As the NPs are exposed to air, new contributions at 75.3 and $72.0 \mathrm{eV}$ appear in the XPS spectrum [Fig. 8(b)] corresponding to the photoemission of $\mathrm{Pt} 4 f$ in $\mathrm{Pt}$ oxide. The estimation of the electron effective-attenuation-length suggests that the electrons collected in the $\mathrm{Pt} 4 f$ XPS experiment come from a depth lower than $0.7 \mathrm{~nm}$. This is an indication that the oxide component should be almost at the surface.

A significant result of this work is the observation of variations in the saturation and the coercivity when the sample is exposed to air. The NPs are left in the glycerin sample holder and measured over a period of days. Since the NPs type is preserved, the observed changes cannot be associated with differences in the NPs, i.e., different sizes, compositions, or interactions. The only agent that seems to be responsible for the observed variations in the magnetic properties is the air exposure of the NPs, as confirmed by XANES, EXAFS, and XPS.

Recent works ${ }^{3-7}$ have proved that the synthesis by a high-boiling coordinating solvent method gives place to a core-shell structure of the FePt NPs. Delalande et al. ${ }^{3}$ have proposed that FePt NPs have a Pt rich core and a Fe rich shell due to the different nucleation kinetics of $\mathrm{Pt}$ and Fe: $\mathrm{Pt}$ nucleates faster and easier than Fe. This is a good picture that could explain the atomic distribution of $\mathrm{Fe}$ and $\mathrm{Pt}$ atoms inside the NPs. Moreover, it agrees well with our XPS, XANES, and EXAFS results that point to a significant amount of oxidized iron and a low Fe-Pt coordination. If Fe nucleates slower than $\mathrm{Pt}$, it is expected to lie at the outmost shell and, therefore, it oxidizes easily. We assume that this exposure originates an oxidation or passiveness of the NPs surface, giving place to a core-shell structure.

The Fe rich shell of FePt NPs is sometimes reported either as nonmagnetic ${ }^{3,5}$ or magnetic. ${ }^{4,6,7}$ Due to the reported $\mathrm{M}_{s}$ values that are much lower than expected for a fcc FePt bulk at $5 \mathrm{~K}$, it is attributed to the presence of the nonmagnetic Fe shell. Nevertheless, the mechanism by which $\mathrm{M}_{s}$ is too low is not completely understood.

Since FePt is an alloy and $\mathrm{M}_{s}$ depends on the iron content, i.e., the higher the iron content the higher the saturation, there is not a determined value of $\mathrm{M}_{s}$ for the equiatomic $\mathrm{FePt}$ like the cases of transition metals $\mathrm{Fe}, \mathrm{Co}$, and Ni. However, we can say that $\mathrm{M}_{s}$ for the equiatomic disordered fcc FePt in bulk is around $80 \mathrm{emu} / \mathrm{g}$ (Ref. 16 and references therein). Taking a glance at Fig. 1, we observe that $\mathrm{M}_{s}$ at $5 \mathrm{~K}$ is about a factor 8 smaller for the FePt NPs and at RT the magnetization at $50 \mathrm{kOe}$ is about $1 \mathrm{emu} / \mathrm{g}$. Similar very low values of $\mathrm{M}_{s}$ have been also reported for "as prepared" FePt NPs. ${ }^{3-5}$

In the case of iron oxide NPs, it is usually reported that the values of $\mathrm{M}_{s}$ are smaller than the bulk values ${ }^{17,18}$ and the behavior follows that the smaller the size is, the smaller the $\mathrm{M}_{s}$. Magnetite NPs synthesized by high temperature decomposition of organic precursors with diameters ranging from 5.7 to $11 \mathrm{~nm}$ have $\mathrm{M}_{s}$ values at $5 \mathrm{~K}$ close to those reported for bulk $\mathrm{Fe}_{3} \mathrm{O}_{4}$; at RT, $\mathrm{M}_{s}$ slightly decreases as the particle diameter decreases. ${ }^{19}$ Martínez et al. ${ }^{18}$ have shown that ther- 
mal decomposition of iron pentacarbonyl in the presence of oleic acid yields a core-shell structure of the type $\mathrm{Fe}_{3} \mathrm{O}_{4} @ \gamma-\mathrm{Fe}_{2} \mathrm{O}_{3}$; despite $\mathrm{M}_{s}$ decreasing with the diameter, $\mathrm{M}_{s}$ of $5 \mathrm{~nm}$ NPs is still about half of the $\mathrm{M}_{s}$ bulk at RT. The open question is why the magnetic saturation is so dramatically reduced in the case of FePt NPs of $4 \mathrm{~nm}$ synthesized by high-boiling coordinating solvent method.

A possible explanation could be the presence of a noncollinear magnetic outmost shell or spin canting that reduces the total magnetic moment of the particle. Magnetic anisotropy rises from the combination of the spin-orbit coupling in the magnetic atom and the lack of spherical symmetry in the electric charge distribution of its environment. Therefore, in NPs, the magnetic anisotropy is an average of bulk $\left(K_{V}\right)$ and surface $\left(K_{S}\right)$ anisotropy that can be approached by $K_{\text {eff }}=K_{V}$ $+(S / V) K_{S}$, with $S$ and $V$ being the surface and the volume of the NP, respectively. Since the FePt NPs are about $4 \mathrm{~nm}$ in size, we can assume they are monodomain; then the estimated effective anisotropy is $K_{\text {eff }}=6 \times 10^{5} \mathrm{~J} / \mathrm{m}^{3}$. In spite of some uncertainties arising from the fact that the calculated value of $K_{\text {eff }}$ does not take into account the size and anisotropy dispersions of the FePt nanoparticles, the magnetic anisotropy is very high and roughly two orders of magnitude larger than the one corresponding to the disordered cubic phase of the FePt bulk $K_{V}=6 \times 10^{3} \mathrm{~J} / \mathrm{m}^{3}$ (Ref. 20), confirming that in FePt NPs the main contribution to the magnetic anisotropy comes from the atoms at the surface.

The $\mathrm{H}_{c}$ for the freshly precipitated sample (batch Ia) is about 1800 Oe. Considering the $K_{V}$ and $\mathrm{M}_{s}$ of the disordered fcc FePt (Refs. 16 and 20) and the density of $\mathrm{Fe}_{45} \mathrm{Pt}_{55}$ (Ref. 21 ), the anisotropy field can be calculated from $\mathrm{H}_{a n i}$ $=2 K_{V} / \mu_{0} \mathrm{M}_{s}$. The obtained value $\mathrm{H}_{a n i}=100 \mathrm{Oe}$, which is the value expected for coherent rotation of single domain $\mathrm{FePt}$ particles and should be close to the $\mathrm{H}_{c}$ for non-interacting monodomain NPs, is a factor 18 smaller than the measured $\mathrm{H}_{c}$.

By using Monte Carlo simulations Labaye et al. ${ }^{22}$ proposed a spin structure for FePt NPs according to the ratio of the surface to the bulk anisotropy. The surface anisotropy, which tries to impose a radial orientation for the surface spins, competes with the bulk anisotropy, which favors the uniaxial alignment of the spins. When the surface anisotropy is much larger than the volume anisotropy (as in our case), this competition gives place to a spin structure with the core spin parallel to each other and the outermost spins radially inward or outward oriented, forming a vortex at the surface of the NPs. This spin configuration has a low net magnetic moment and a large magnetic hysteresis. Therefore, the spin structure with a vortex at the surface can explain both the very low $\mathrm{M}_{s}$ and the surprisingly high $\mathrm{H}_{c}$.

The exposition to air of the nonoxidized "as made" FePt NPs reduces $\mathrm{M}_{s}$ and $\mathrm{H}_{c}$ as well. This can be explained by the rapid formation of an oxide shell giving place to a structure like FePt @ $\mathrm{Fe}_{x} \mathrm{O}_{y}$. The natural way Fe oxidizes is $\gamma-\mathrm{Fe}_{2} \mathrm{O}_{3}$ (ferrimagnetic) or $\alpha-\mathrm{Fe}_{2} \mathrm{O}_{3}$ (anti-ferromagnetic); from both kinds of oxides the former is the most probable. However, the XANES measurements at $\mathrm{Fe}-\mathrm{L}_{2,3^{-}}$and $\mathrm{K}$-edges confirms the oxidation state of Fe comes close to the $\alpha-\mathrm{Fe}_{2} \mathrm{O}_{3}$ (hematite), although the thickness of the shell that ranges from 0.3 (from SQUID magnetometry) to $0.7 \mathrm{~nm}$ (from XPS) is small enough to be a highly disordered structure. By comparing batch Ia with batch IIa (compare Fig. 1 with Fig. 3) it is possible to observe that after oxidation the hysteresis loop of batch IIa is open even at $50 \mathrm{kOe}$, which is characteristic for anti-ferromagnetic behavior.

The formation of hematite at the surface, which is antiferromagnetic, bears dramatically on $\mathrm{H}_{c}$, reducing it from 1400 Oe to 300 Oe after 30 days air exposure. Since the outmost shell is Pt-depleted, the shell is not a pure hematite; it is hematite with a cluster of $\mathrm{Pt}$ atoms. The magnetic properties of an anti-ferromagnet is strongly affected by the presence of impurities. ${ }^{23}$ Despite the magnetic anisotropy of the hematite being high, it tends, according to the observation, to soften the original spin structure because the anisotropy of this hematite shell with Pt cluster is evidently softer than the nonoxidized spin structure. To enlighten this, let the spins of the nonoxidized FePt all point radially. This spin configuration has the maximum magnetic anisotropy (although the total magnetic moment is null): if the oxidation of the surface makes the spins move away from radial orientation, the spin configuration is less anisotropic than the former one, inducing a softening of the overall magnetic anisotropy.

Since the shell formed at the surface is antiferromagnetic, it must interact with the ferromagnetic core when the sample is cooled down in an applied field. Hysteresis loop measurements were performed according to the ZFC-FC procedure at applied field $50 \mathrm{kOe}$. As can be seen from Fig. 4, the FC hysteresis loop is wider than the ZFC one and it is also shifted. Since exchange interactions at the interface between a ferromagnet (FM) and an antiferromagnet (AFM) may create a preferential direction for the spin orientation of the $\mathrm{FM},{ }^{24-26}$ the widening of the hysteresis loop reflects the exchange coupling at the core-shell. If the anisotropy of the AFM phase is not large enough, as in this case, the exchange coupling at the interface makes it such that the necessary energy to rotate the magnetic moments of the FM is greater than in the absence of interfaces, resulting in an increase of the coercivity. The observation of an exchange bias of 60 Oe could be due to the shell thickness. The surface region exhibits modified magnetic properties compared to the bulk; as a consequence of lacking structural periodicity and competing magnetic interactions, ${ }^{27,28}$ such coexistence of topological disorder and frustration of magnetic interactions has already been reported for metallic systems. $^{29,30}$

\section{SUMMARY}

In summary, we report here that $4 \mathrm{~nm} \mathrm{FePt} \mathrm{NPs} \mathrm{can}$ oxidize when they are exposed to air, forming a core-shell structure. According to the XANES and EXAFS results, the oxidation state of $\mathrm{Fe}$ comes close to the hematite, although the oxidized surface is likely disordered and magnetically frustrated. XPS measurements at Pt $4 f$ show that after some days of air exposure Pt also oxidizes. The coercivity is mainly affected by the formation of the shell, being reduced to one third of the original value; the oxide shell bears also on $\mathrm{M}_{s}$. The shell is anti-ferromagnetic and interacts with the 
inner part of the NPs when the sample is cooled in an external applied field of $50 \mathrm{kOe}$. The interaction gives place to an increase of the coercivity and an exchange bias of $60 \mathrm{Oe}$. However, despite this interaction, the oxidized FePt is softer than the "as made" one.

\section{ACKNOWLEDGMENTS}

This work was financially supported by CM (Grant No. S-0505/MAT/0194) and MCyT (Grant Nos. MAT200506119 and NAN2004-09125-C07-05), Spain; ANPCYT (Grant Nos. PICT 06-17492 and 25515) and CONICET (PIP 6075), Argentina; and LNLS (Project No. D04B-XAFS16609), Campinas, Brazil. One of the authors (P.d.1.P.) gratefully acknowledges S. J. Stewart for the critical reading of the manuscript.

${ }^{1}$ P. Mulvaney, MRS Bull. 26, 1009 (2001).

${ }^{2}$ S. Sun, Adv. Mater. 18, 393 (2006).

${ }^{3}$ M. Delalande, P. R. Marcoux, P. Reiss, and Y. Samson, J. Mater. Chem. 17, 1579 (2007).

${ }^{4}$ H. L. Wang, Y. Huang, Y. Zhang, G. C. Hadjipanayis, D. Weller, and A. Simopoulos, J. Magn. Magn. Mater. 310, 22 (2007).

${ }^{5}$ X. W. Wu, C. Liu, L. Li, P. Jones, R. W. Chantrell, and D. Weller, J. Appl. Phys. 95, 6810 (2004)

${ }^{6}$ S. Anders, M. F. Toney, T. Thomson, J.-U. Thiele, B. D. Therris, S. Sun, and C. B. Murray, J. Appl. Phys. 93, 7343 (2003).

${ }^{7}$ B. Stahl, J. Ellrich, R. Theissmann, M. Ghafari, S. Bhattacharya, H. Hahn, N. S. Gajbhiye, D. Kramer, R. N. Viswanath, J. Weissmüller, and H. Gleiter, Phys. Rev. B 67, 014422 (2003).

${ }^{8}$ H. Zeng, J. Li, J. P. Liu, Z. L. Wang, and S. Sun, Nature (London) 420 395 (2002)

${ }^{9}$ J. Li, Z. L. Wang, H. Zeng, S. Sun, and J. P. Liu, Appl. Phys. Lett. 82, 3743 (2003).

${ }^{10}$ S. Sun, C. B. Murray, D. Weller, L. Folks, and A. Moser, Science 287, 1989 (2000).

${ }^{11}$ P. de la Presa, M. Multigner, M. P. Morales, T. Rueda, E. Fernández-Pinel, and A. Hernando, J. Magn. Magn. Mater. 316, e753 (2007).

${ }^{12}$ B. Ravel and M. Newville, J. Synchrotron Radiat. 12, 537 (2005).

${ }^{13}$ S. I. Zabinsky, J. J. Rehr, A. Ankudinov, R. C. Albers, and M. J. Eller, Phys. Rev. B 52, 2995 (1995).

${ }^{14}$ C. J. Powell and A. Jablonski, NIST Electron Effective-Attenuation-Length Database-Version 1.1 (National Institute of Standards and Technology, Gaithersburg, MD, 2003).

${ }^{15}$ S. Anders, S. Sun, C. B. Murray, C. T. Rettner, M. E. Best, T. Thomson, M. Albrecht, J.-U. Thiele, E. E. Fullerton, and B. D. Terris, Microelectron. Eng. 61-62, 569 (2002)

${ }^{16}$ J. Lyubina, "Nanocrystalline Fe-Pt alloys: Phase transformations, structure and magnetism," dissertation, Dresden, 2007, Cuvillier Verlag, Germany, ISBN-10: 3867272336 .

${ }^{17}$ D. L. Huber, Small 1, 482 (2005).

${ }^{18}$ C. Martínez-Boubeta, K. Simeonidis, M. Angelakeris, N. Pazos-Pérez, M. Giersig, A. Delimitis, L. Nalbandian, V. Alexandrakis, and D. Niarchos, Phys. Rev. B 74, 054430 (2006).

${ }^{19}$ A. G. Roca, M. P. Morales, K. O'Grady, and C. J. Serna, Nanotechnology 17, 2783 (2006).

${ }^{20}$ V. G. Pynko, A. S. Komalov, and L. V. Ivaeva, Phys. Status Solidi A 63, K127 (1981).

${ }^{21}$ O. A. Ivanov, L. V. Solina, V. A. Demshina, and L. M. Magat, Fiz. Met. Metalloved. 35, 92 (1973).

${ }^{22}$ Y. Labaye, O. Crisan, L. Berger, J. M. Greneche, and J. M. D. Coey, J. Appl. Phys. 91, 8715 (2002).

${ }^{23}$ S. Mørup, D. E. Madsen, C. Frandsen, C. R. H. Bahl, and M. F. Hansen, J. Phys.: Condens. Matter 19, 213202 (2007).

${ }^{24}$ R. H. Kodama and A. E. Berkowitz, Phys. Rev. B 59, 6321 (1999).

${ }^{25}$ J. Nogués and I. K. Schuller, J. Magn. Magn. Mater. 192, 203 (1999).

${ }^{26}$ J. Nogués, J. Sort, V. Langlais, V. Skumryev, S. Suriñach, J. S. Muñoz, and M. D. Baró, Phys. Rep. 422, 65 (2005).

${ }^{27}$ B. Martínez, X. Obradors, L. Balcells, A. Rouanet, and C. Monty, Phys. Rev. Lett. 80, 181 (1998).

${ }^{28}$ E. Tronc, A. Ezzir, R. Cherkaoui, C. Chanéac, M. Nogués, H. Kachkachi, D. Fiorani, A. M. Testa, J. M. Grenèche, and J. P. Jolivet, J. Magn. Magn. Mater. 221, 63 (2000)

${ }^{29}$ A. Hernando, E. Navarro, M. Multigner, A. R. Yavari, D. Fiorani, M. Rosenberg, G. Filoti, and R. Caciuffo, Phys. Rev. B 58, 5181 (1998).

${ }^{30}$ E. Bonetti, L. Del Bianco, D. Fiorani, D. Rinaldi, R. Caciuffo, and A. Hernando, Phys. Rev. Lett. 83, 2829 (1999). 